\title{
La mediación pedagógica de un modelo de aprendizaje centrado en el estudiante. Implicaciones en la unidad didáctica impresa, estudiante y profesor, en la Universidad Estatal a Distancia de Costa Rica
}

\author{
Xinia María Calvo Cruz* \& Victor Hugo Méndez-Estrada** \\ Recibido: febrero 2010 • Aceptado: abril 2011
}

\begin{abstract}
RESUMEN
El propósito es brindar elementos teórico-prácticos para la discusión, a lo interno de la UNED y a lo externo, acerca de los fundamentos que deben caracterizar a un modelo pedagógico de educación a distancia centrado en el estudiante. Se plantean apuntes teóricos que respaldan la necesidad social de la educación a distancia y focaliza su atención en la responsabilidad que se le atribuye al estudiante como centro del modelo pedagógico de la UNED, así como el lugar de privilegio del texto impreso como mediación en los procesos de enseñanza y aprendizaje a distancia. Se ofrece una propuesta de competencias de los mediadores pedagógicos en un aprendizaje mediatizado en la educación a distancia.
\end{abstract}

Palabras claves: unidad didáctica, aprendizaje autónomo, modelo pedagógico, competencias, centrado en el estudiante.

\section{ABSTRACT}

The purpose is to bring elements, in theory and practice, to the discussion, both within the UNED and from external sources, about the fundamentals that should characterize

* Xinia María Calvo Cruz. UNED, PACE. Magister en Planificación Educativa y Licenciada en Educación Primaria I y II Ciclo de la Universidad de Costa Rica. Profesora Universitaria de la Universidad de Costa Rica de cursos de Currículum y Pedagogía y de Universidades privadas en diversidad de cursos afines al Diseño Curricular, Artes del Idioma, Didáctica general y específica, Investigación, Experiencia profesional docente, entre otros.xcalvo@uned.ac.cr

** Víctor Hugo Méndez-Estrada. UNED, PROIFED. Biólogo y Magister Scientiae en Educación con énfasis en investigación. Labora para la UNED desde 1979, como profesor e investigador. En otras universidades ha sido profesor de Biología, Genética y de Métodos de investigación. Cuenta con 60 publicaciones entre artículos y libros.mendezv@uned.ac.cr 
a pedagogical model of distance education centered on the student. We suggest some theoretical insights that support the social need of distance education and focus their attention on the responsibility attributed to the student as the core of the pedagogical model in UNED, as well as the privileged place that printed material has as a medium on the processes of distance teaching and learning. We offer a proposal of the skills the pedagogical mediators should have on media-oriented learning for distance education.

Key words: teaching unit, autonomous learning, pedagogical model, competencies.

\section{Presentación}

En el XV Congreso de Tecnología y Educación a Distancia de la UNED, se afirma que "Las nuevas condiciones mundiales, marcadas por la incertidumbre, la globalización, la tecnologización de los procesos y las tecnologías para la comunicación -que han reducido las barreras de tiempo y de espacio-, tienden a desdibujar las fronteras entre diferentes modalidades educativas" y hacer de la educación un proceso para que el ser humano logre transformar y adaptarse a una sociedad del conocimiento gobernada por la racionalidad científica y tecnológica (Cardona, 2002) que transforma el modo de pensar, de sentir y de actuar de cada uno. Desde esa perspectiva, la educación es la llamada a brindar las competencias que exige este tipo de sociedad; por lo tanto, el modelo pedagógico ha determinado cuál sistema educativo debe considerar el currículo y las competencias necesarias para que el estudiante logre adaptarse y transformar su entorno. Estos son los retos que asumen los sistemas educativos a distancia.

Los objetivos procuran brindar elementos teórico-prácticos para la discusión, a lo interno de la UNED y a lo externo, acerca de los fundamentos que deben caracterizar a un modelo pedagógico de educación a distancia centrado en el estudiante, y analizar sus implicaciones al elaborar las unidades didácticas modulares como mediadoras del aprendizaje.

\section{Metodología}

Para poder cumplir con los objetivos, primero se realiza una revisión bibliográfica, seguida de una discusión teórico analítica y crítica de los fundamentos científicos y filosóficos que caracterizan a un modelo pedagógico de educación a distancia centrado en el estudiante. Luego se hace un análisis bibliográfico de 287 documentos impresos y digitales, producto de las investigaciones realizadas en la UNED, durante 1989 al 2010 en cinco centros creados para ese fin (Méndez y 
Aguirre, 2011), para recoger información relacionada con los medios y recursos que se pueden incluir para la elaboración de una unidad didáctica modular, como mediatizadora en la enseñanza y aprendizaje a distancia.

\section{La formación a distancia: una respuesta a las necesidades sociales. Breves reflexiones teóricas}

El auge de la educación a distancia es tema de conversación en muchos espacios que buscan respuestas a las necesidades de la sociedad actual (García, 2000; Berrocal, 2009; Brenes, 2009). Son diversas las razones que se proponen para explicar el incremento de esta forma de educar. Se citan como primordiales y recurrentes tres de ellas: el aumento de la demanda de la sociedad misma, puesto que no es nuevo y a lo largo de su existencia se han visto las bondades de la misma; además se cita la imperiosa necesidad económica de reducir costos en la educación (Mayorga, 1999; Morales, 2006) y, finalmente, sin duda alguna, la introducción de las tecnologías de comunicación en los diferentes sectores, expandiendo las múltiples aplicaciones a contextos educativos (Laborí, s.f.; Méndez et al., 2004; Monge, 2004; Berrocal, 2009; Brenes, 2009).
En una sociedad tan convulsa como la de hoy, la estructura de horarios de la educación presencial contrasta con las necesidades reales de los estudiantes, razón por la cual la practicidad que ofrece la distancia ha generado un salto importante, por lo que poco a poco se va difuminando la idea de la dificultad de aprender sin un profesor al frente ofreciendo su saber, sin una rutina de horario y en ausencia de cuatro paredes que presencien la construcción del conocimiento.

En este contexto, el aprendizaje a distancia, atribuye el papel protagónico al estudiante, puesto que le demanda un proceso personal y autónomo. Lo anterior, le exige poseer conocimiento de sí mismo, conocer su estilo y ritmo de aprendizaje y programar sus periodos de estudio con su vida cotidiana. Valga aclarar que esto último demanda algunas competencias que más adelante se esbozarán.

La educación a distancia coloca más peso sobre el estudiante que la modalidad presencial porque prioriza el aprendizaje autónomo, autodirigido $\mathrm{y}$ autorregulado $\mathrm{y}$ considera al estudiante como ente responsable y gestor de su aprendizaje (Brockett y Hiemstra, 1993; Duart, 2000); de acá que la responsabilidad recae en él y el docente pasa de un rol directivo a ser un guía y orientador (Cabrera et al., 
2004) y en definitiva el avance es responsabilidad del estudiante.

El término "aprendizaje autodirigido" $\mathrm{u}$ otros casi equivalentes como "autoaprendizaje" o "aprendizaje autónomo", se pueden entender grosso modo como la posibilidad que se le otorga al estudiante de forjar sus propios instrumentos y hábitos de aprendizaje; es decir, que cuenta con "la libertad de aprovechar al máximo el apoyo que se le ofrece, de planificar su progreso de aprendizaje universitario y de regular su propio ritmo de trabajo." (Duart, 2000: 5)

Contreras (1999) plantea que la autonomía se refiere a la capacidad de una persona para elegir lo que es valioso para él, es decir, para realizar elecciones en sintonía con su autorrealización. El aprendizaje independiente es el proceso de desarrollo de las habilidades cognitivas psicomotoras y de valores en que el estudiante, o más bien el sujeto cognoscente, asume por completo la responsabilidad de su propio aprendizaje. Esto significa tomar la iniciativa de diagnosticar sus necesidades educativas, elegir y poner en práctica estrategias de aprendizaje idóneas para el logro de las metas propuestas y autorregular el aprendizaje. Exige el desarrollo de capacidades personales en las actitudes, las habilidades, los saberes y los procedimientos.
Se deduce una diferencia entre aprendizaje "independiente" y "autónomo". El aprendizaje autónomo es un proceso que requiere que la persona sea autor de su propio desarrollo -al igual que en el aprendizaje independiente-; sin embargo, este proceso ocurre dentro de un marco institucional que le permite al educando elegir los caminos, las estrategias, las herramientas y los momentos que considere pertinentes para aprender y poner en práctica lo que ha aprendido. Es una forma de aprendizaje en que la persona y la institución buscan potenciar en conjunto la transformación y el cambio.

Brockett y Hiemtra (1993: 24) desarrollan el concepto de aprendizaje autodirigido como "... una combinación de fuerzas tanto interiores como exteriores de la persona que subrayan la aceptación por parte del estudiante de una responsabilidad cada vez mayor respecto a las decisiones asociadas al proceso de aprendizaje".

En el aprendizaje independiente/autónomo/autodirigido, el estudiante debe ser capaz tanto de identificar sus necesidades de aprendizaje como de satisfacerlas. Así, el aprendizaje no se limita a las horas presenciales que se pasan en la institución educativa, ya que los límites de ésta se amplían a múltiples escenarios (trabajo, casa, oficina, centro penal, entre otros). 


\section{Aprendizaje en el nivel universitario}

El aprendizaje en el espacio universitario supone unas particularidades especiales: se da en sujetos con un repertorio previo de conocimientos acumulado y organizado cognitivamente según los cánones pedagógicos de los niveles educativos anteriores. Esto puede facilitar o entorpecer los nuevos aprendizajes universitarios centrados en el estudiante según sean el nivel de continuidad y "encaje" pedagógico con el nuevo contexto educativo superior. Supone que los sujetos de aprendizaje ya poseen estructuras cognitivas que pueden permitir la reflexividad, creatividad y análisis de los diversos conocimientos. Además, el aprendizaje universitario no es una experiencia pasiva y reproductiva sino constructiva y holista, capaz de generar espacios cognitivos, individuales y sociales de reflexión, diálogo epistémico, creatividad, análisis e integración de los diversos saberes profesionales. Por lo tanto, la universidad no puede ser un espacio en donde el conocimiento únicamente sea transmisible y el aprendizaje sea pasivo; muy por el contrario, el profesional que se forma en los ambientes universitarios debe poder llegar a ser una persona con una serie de competencias y habilidades de aprendizaje que le permita seguir generando conocimientos aun fuera de las universidades.

Los aspectos anteriores han sido el sustento teórico para el quehacer académico de la Universidad Estatal a Distancia -UNED- de Costa Rica.

\section{Los lineamientos del modelo pedagógico de la universidad estatal a distancia (uned) están centrados en el estudiante}

Desde la creación de la UNED, en 1977, se concibió a los medios de comunicación social como importantes para la mediación pedagógica (UNED, 2007). En su Ley de creación (Ramírez, 2006: 130) se señala, en el artículo 2, como objetivos institucionales, los siguientes:

a. Fortalecer los valores en que está fundado el Estado costarricense;

b. Proporcionar educación superior mediante la utilización de técnicas de comunicación social;

c. Incorporar a la educación superior, con métodos idóneos y flexibles, a quienes no hubieren podido incorporarse al sistema formal universitario;

e. Proporcionar instrumentos adecuados para el perfeccionamiento y formación permanente de todos los habitantes; 
f. Servir de vehículo para la difusión de la cultura;

i. Fomentar el espíritu científico, artístico, cultural y cívico del pueblo costarricense.

Para el logro de sus objetivos de creación, se han establecido lineamientos institucionales para un modelo pedagógico centrado en el estudiante que sea capaz de

"permitirle, a éste, la libertad de aprovechar al máximo los recursos que se le ofrecen, de planificar el progreso de su aprendizaje y de regular, él mismo, el ritmo y la calidad de sus avances. Esto implica que todos los elementos del modelo pedagógico se piensen para ponerlos a disposición de los estudiantes, de manera que ellos puedan gestionar su propio proceso de formación". (UNED, 2007: 28)

En los lineamientos del modelo pedagógico, el estudiante es el gestor de su propio aprendizaje (aprendizaje autodirigido). En otras palabras, formula guías para renovar las estrategias didácticas, los materiales de estudio, la evaluación del aprendizaje y los apoyos que se ofrecen al estudiante para lograr esta meta. Sin embargo, el reconocer que el modelo propone que la experiencia educativa se centre en el estudiante, no agota el tema; muy por el contrario, nos encara con el desafío de construir propuestas, modelos y estrategias para alcanzar tal ideal.

Los principios y fundamentos del Modelo Pedagógico de la UNED cubren prácticamente todos los elementos curriculares: estrategias didácticas, los materiales, la evaluación, el profesor y la planificación. Sin embargo, indicarlo en papel es más simple que operacionalizarlo y verlo en la práctica del acto educativo.

En este sentido, Méndez et al. (2008) en su propuesta de trabajo titulado "Los modelos pedagógicos centrados en el estudiante; apuntes sobre los procesos de aprendizaje y enseñanza", hacen una serie de reflexiones que tienen como fin la generación de elementos de juicio, que como fruto de la autocrítica y reflexión consensuada, ayuden a los docentes de la UNED a diseñar aplicaciones de la pedagogía basada en el estudiante ya sea como sujeto u objeto del acto educativo.

Así, entonces, es de interés considerar las reflexiones citadas, en aras de clarificar las implicaciones de un modelo pedagógico centrado en el estudiante. Al respecto, se clarifica que las universidades apuntan hacia la definición de un modelo pedagógico con el fin de tener una guía sobre el acto educativo, que considera las formas de intervención.

Para la UNED el Modelo Pedagógico debe "contener un 
conjunto de principios, normas y criterios que orientan cada una de las actividades de enseñanza y de aprendizaje que se emprenden en la ejecución práctica del proyecto de educación universitaria a distancia." (UNED, 2007: 15). Es un conjunto ideal de principios rectores del quehacer pedagógico que considera, como fuentes, la propia historia como institución a distancia, pionera a nivel nacional y de América Latina; en segundo lugar, las posturas teóricas de mayor significancia para la comprensión y conducción de la educación a distancia y finalmente se nutre de todas las disciplinas que en la actualidad se relacionan con el estudio del conocimiento humano.

En un afán por ubicar el modelo de la UNED, Méndez et al. (2008), en su análisis plantean tres macro modelos pedagógicos: el conductista, el romántico o naturalista y el constructivista. Para cada uno de ellos se analizan los fundamentos, orígenes, lineamientos educativos, evaluación crítica, fortalezas y debilidades, así como las áreas en las cuales el modelo podría ser aplicado.

Como parte de las consideraciones finales, el documento de Méndez et al. (2008) plantea la necesidad de peguntarse si la UNED cumple con las expectativas de un modelo pedagógico, así como si es pertinente adoptar alguno de los modelos pedagógicos abordados, sin obviar la claridad que se debe tener en cuanto a las limitaciones de cada uno o las implicaciones de la combinación de algunos.

Además, queda claro que cada una de estas líneas que marcan el protagonismo del estudiante responde a una concepción muy distinta de ser humano y que, por tanto, las decisiones deben estar enmarcadas por la pedagogía y la epistemología; pero sí con mediaciones didácticas tradicionales y de avanzada.

\section{Los medios de comunicación social como mediadores de la enseñanza a distancia centrada en el estudiante}

En las políticas institucionales se señala que "desde la descripción curricular hasta el modelo instruccional, se da una atención focalizada en el autoaprendizaje del estudiante. Así, en el sistema a distancia, el material escrito es el medio principal de enseñanzaaprendizaje, el cual se denomina "Unidad didáctica" (PROCUR, 1992: 7). Al ser protagónicos los medios para la consecución de sus logros, su gestión se orienta hacia la producción de los materiales didácticos escritos, apoyados con tutorías presenciales y virtuales, con materiales audiovisuales y con 
tecnologías de la información y comunicación, considerados complementarios al escrito en el proceso de enseñanza y aprendizaje (Monge et al., 1999; Berrocal, 2009). Esto por cuanto para la UNED, los nuevos tiempos requieren de una "concepción metodológica más abierta, más flexible, que ofrezca, al estudiante las herramientas para construir su propio proceso de aprendizaje y lo haga protagonista en la construcción del conocimiento" (UNED, 2007: 11).

Se deduce de las políticas institucionales dos elementos claves: la atención focalizada al autoaprendizaje del estudiante y la unidad didáctica modular como medio principal en el sistema a distancia.

Ahora bien, si se parte del hecho de que el modelo pedagógico de la UNED está centrado en el estudiante, será necesario clarificar qué implicaciones se tiene en los materiales, en este caso específicamente la unidad didáctica, y en los demás actores del proceso educativo: el profesor y el estudiante.

\section{La unidad didáctica como parte de la unidad didáctica modular. Competencias de los actores. Relevancia de la situación}

La educación a distancia se caracteriza por la separación física en tiempo y espacio, entre el estudiante y el profesor, y porque requiere de una comunicación mediatizada por parte de los medios de comunicación social, así como por la responsabilidad de autogestión que asume el estudiante durante el proceso de aprendizaje.

En la actualidad, en la UNED el diálogo mediado entre profesor y estudiante se logra a través de la unidad didáctica modular (UDM), que Láscaris (2005) define como una unidad específica compuesta por los diversos recursos y materiales didácticos cohesivos e integrados didácticamente para facilitar el aprendizaje a distancia.

En esa visión se infiere que el profesor y estudiante tienen roles en torno a la UDM y que, por tanto, se requieren competencias que les permitan asumir dichos roles.

Al respecto, García (2000) indica que este sistema exige responsabilidades en la configuración y funciones de los equipos académicos que se convierten en quienes planean todas las etapas y en las cuales la interdependencia es un factor que no se puede hacer a un lado.

Para efectos del presente artículo es básico considerar la importancia que han tenido las competencias de los mediadores pedagógicos del proceso educativo, donde se hace prioritario considerar que el modelo pedagógico de la UNED está centrado en el 
estudiante y ello acarrea implicaciones para el trabajo con la unidad didáctica modular.

El avance tecnológico no ha restado el liderazgo al medio impreso como soporte de la enseñanza en el mundo; por el contrario, ha favorecido los procesos de producción de este medio de comunicación social.

Al ser la educación a distancia el marco de este escrito, es válido destacar el hecho de la utilización del texto como base de la formación. En el caso de la UNED, este medio ocupa la base de la unidad didáctica modular. Lo anterior no excluye las bondades de los otros recursos, como los son las videoconferencias, los multimedios o las innumerables opciones que ofrecen las plataformas virtuales (Milachay, 2007; Berrocal, 2009; Brenes, 2009), pero es un hecho que el impreso sigue manteniendo su lugar de privilegio.

Sobre la respuesta a la pregunta ¿cuáles son las razones por las que el impreso ocupa un lugar tan importante en la formación a distancia?, se pueden citar las siguientes (Trilla et al., 2003):

- un medio muy accesible, sin importar que se trate de un libro, cuaderno u otra presentación, ya que el impreso es de fácil manipulación. Además se puede consultar en cualquier espacio y condición al ritmo individual del lector.

- es un medio productivo, de fácil reproducción. En este aspecto, es válido destacar que en la UNED la producción del texto pedagógico genera largos tiempos de espera en el proceso de su diseño y producción, situación que está en la mira institucional y que se proyecta como un tema importante del IV congreso universitario por realizarse en el 2011.

- $\quad$ es un medio flexible, ya que el impreso tiene la utilidad que se le quiera dar: lectura base, medio de consulta; también la intención del lector es variable, pues puede hacer anotaciones o incluso subrayar las ideas expuestas.

- tradición libresca: su aporte es básico desde los inicios de la educación a distancia.

- un medio relativamente poco costoso: tanto para el estudiante como para la producción, si se compara con la utilidad del mismo.

Las anteriores características hacen del texto impreso un medio por excelencia en la educación a distancia, pero el mismo autor señala limitantes importantes que deben ser consideradas.

- un medio lineal: utiliza un lenguaje un poco reductor que 
trata las relaciones complejas "a la carrera" con una lógica elemental,

- poco sensible a las diferentes culturas: a pesar de ser un medio accesible y democrático, se asume como una experiencia desigual en función del medio socio-cultural del lector,

- poco interactivo: en la formación a distancia se realizan esfuerzos sistemáticos para favorecer el diálogo profesorestudiante al interior de los textos pedagógicos; sin embargo, lograr el diálogo por un medio impreso no es tarea sencilla, razón por la cual se piensa en que el texto simula una cierta interacción (de manera muy limitada en comparación con otros medios, que sí pueden ofrecer este espacio).

Consientes de estas limitaciones, en la UNED se realizan esfuerzos tendientes a complementar el medio escrito con otros recursos didácticos: laboratorios virtuales, plataformas virtuales, correo electrónico, videoconferencias y audiovisuales, entre otros. Recursos que se han implementado he investigado con el propósito de analizar su potencial interactivo para la construcción de conocimientos en un sistema de enseñanza y aprendizaje a distancia cuyo modelo está centrado en el estudiante.
VI. Competencias básicas de los actores de los procesos de enseñanza y aprendizaje a distancia; sus implicaciones para la elaboración de unidades didácticas modulares (UDM) como mediadoras del aprendizaje

Entendida la UDM como el medio escrito complementado con tecnologías de la información y comunicación, se hace necesario analizar los esfuerzos que se han realizado en la UNED para convertirla en el recurso principal para la mediación pedagógica.

El análisis de las 287 investigaciones realizadas en la UNED entre 1989 y 2010 , de las cuales se encuentran digitales o en medio impreso, se encontró que 216 tienen relación con la mediación pedagógica de un modelo de aprendizaje centrado en el estudiante: unidad didáctica impresa, estudiante $\mathrm{y}$ profesor (Cuadro 1).

El uso de la tecnología como mediador de los procesos de enseñanza y aprendizaje a distancia conlleva implicaciones al elaborar las unidades didácticas modulares (UDM), pues el material impreso se verá complementado con las mismas. Entre la plataforma tecnológica empleada se encuentra el correo electrónico (Cruz et al., 2004; D’Agustino et al., 2006); audioconferencia (Cruz et al., 2004); 
CUADRO 1

Frecuencia por temática de los estudios realizados en los distintos centros de investigación de la UNED. 1989-2010

\begin{tabular}{lcc}
\multicolumn{1}{c}{ Temática } & f & $\%$ \\
Uso de la tecnología en la educación a distancia & 86 & 40 \\
Desarrollo educativo a distancia & 35 & 16 \\
Evaluación de los aprendizajes & 21 & 10 \\
Evaluación del material impreso & 19 & 9 \\
Diseño curricular & 16 & 7 \\
Deserción estudiantil & 14 & 6 \\
Enseñanza y aprendizaje a distancia & 12 & 6 \\
Vida estudiantil & 11 & 5 \\
Autoevaluación de carreras & 2 & 1 \\
TOTAL & 216 & 100 \\
\hline
\end{tabular}

videoconferencia (D'Agustino et al., 2006; Méndez y Monge, 2006); webb (Monge-Nájera y Aguilar, 1999); Internet (Monge-Nájera et al., 2001); fax (D’Agustino et al., 2006); Learning Space (Gutiérrez et al., 1998); toolbook (Méndez, 1998); Quórum (Méndez, 1999); microcampus (Méndez y Monge, 2006); TICs (Méndez \& Monge, 2006) y laboratorios virtuales (Méndez \& Monge, 2004; Monge \& Méndez, 2007). También existen 11 investigaciones $(6 \%)$ que tienen que ver con los procesos de enseñanza y aprendizaje a distancia, donde se analizan las competencias en la lectura y escritura que deben tener los estudiantes para adquirir nuevos conceptos en una modalidad de enseñanza y aprendizaje a distancia cuyo mediador principal es el texto escrito (Rodino, 1999).
A la luz del marco de la educación a distancia que realiza la UNED, con lineamientos de un modelo pedagógico centrado en el estudiante y una unidad impresa -apoyada por las tecnologías de la información y comunicación- como base de la unidad didáctica modular, es fundamental preguntarse ¿cuáles son las competencias necesarias de al menos los tres elementos curriculares que soportan la metodología a distancia: estudiante, profesor y materiales didácticos?

En un intento por resumir ideas de la literatura en torno a esta temática y la experiencia de los autores en la UNED, a continuación se presenta un cuadro que sintetiza las competencias laborales (básicas) requeridas por los actores sociales del acto educativo a distancia (Cuadro 2). 


\section{CUADRO 2}

Competencias laborales de los mediadores pedagógicos, estudiante y profesor, en un aprendizaje mediatizado para la UNED.

\begin{tabular}{|c|c|c|}
\hline COMPETENCIAS & ESTUDIANTE & PROFESOR \\
\hline COMUNICATIVAS & $\begin{array}{l}\text { Poseer un buen manejo del } \\
\text { lenguaje, que le permita } \\
\text { un mensaje fluido en sus } \\
\text { trabajos y construcción del } \\
\text { conocimiento con profesor y } \\
\text { compañeros. }\end{array}$ & $\begin{array}{l}\text { Poseer un buen manejo del } \\
\text { lenguaje, que le permita un } \\
\text { mensaje fluido y cordial con el } \\
\text { estudiante. }\end{array}$ \\
\hline TECNOLÓGICAS & $\begin{array}{l}\text {-utilizar eficaz y } \\
\text { adecuadamente la } \\
\text { computadora, Internet } \\
\text { y los diferentes recursos } \\
\text { tecnológicos. } \\
\text {-participar en espacios de } \\
\text { construcción de conocimiento } \\
\text { sincrónicos y asincrónicos. }\end{array}$ & $\begin{array}{l}\text {-utilizar eficaz y adecuadamente } \\
\text { la computadora, Internet y demás } \\
\text { recursos tecnológicos. } \\
\text {-liderar espacios de construcción } \\
\text { de conocimiento, tanto } \\
\text { sincrónicos como asincrónicos } \\
\text { (foros, Chat, debates). } \\
\text {-fortalecer las comunidades de } \\
\text { aprendizaje. }\end{array}$ \\
\hline $\begin{array}{l}\text { GESTIÓN } \\
\text { EDUCATIVA }\end{array}$ & $\begin{array}{l}\text {-ser independiente y autodirigir } \\
\text { su proceso formativo, es decir } \\
\text { autogestionar su aprendizaje. } \\
\text {-regular el proceso de aprender } \\
\text {-construir, resumir y recopilar } \\
\text { información. } \\
\text { - realizar trabajo colaborativo } \\
\text { - con espíritu creativo y de } \\
\text { investigación }\end{array}$ & $\begin{array}{l}\text { - facilitar el aprendizaje } \\
\text {-guiar y dar seguimiento y } \\
\text { atención a lo largo del proceso } \\
\text { formativo (regular el proceso) } \\
\text {-incentivar la formación de } \\
\text { alumnos críticos y de pensamiento } \\
\text { creativo } \\
\text {-incentivar la autorregulación del } \\
\text { aprendizaje } \\
\text {-incorporar el trabajo colaborador } \\
\text { entre estudiantes. } \\
\text {-elaborar materiales didácticos } \\
\text { que complementen la unidad } \\
\text { didáctica modular. } \\
\text {-evaluar el proceso y el producto } \\
\text { de acuerdo con las circunstancias } \\
\text { y necesidades. } \\
\text {-propiciar espacios de } \\
\text { investigación como parte del } \\
\text { mejoramiento continuo. }\end{array}$ \\
\hline
\end{tabular}

Fuente: Elaborado por Xinia María Calvo Cruz y Víctor Hugo Méndez Estrada. 
Esas competencias ya han sido abordas en forma individual por investigadores de la UNED, específicamente las relacionadas con las TIC y la lectoescritura (Rodino, 1999).

Ahora bien, con respecto a la unidad didáctica modular, se hace énfasis en el texto impreso. Al respecto se cita como competencias de este medio las siguientes:

- tener claridad en su estructura y dosificación de contenido

- promotor de diversidad de líneas de pensamiento con respecto al contenido

- presentar tratamiento progresivo en las temáticas

- utilizar un lenguaje acorde con la diversidad del estudiante que puede tener acceso al medio impreso.

- involucrar experiencias de autorregulación que permitan simular un diálogo constructivista entre el autor y el estudiante.

- poseer un discurso expositivo de calidad (estilo sobrio y académico)

- Diseñar de forma agradable a la vista del lector (tipo e letra, espacios, recursos ilustrativos, entre otros) (Monge et al. 2009)

\section{Etapas del material impreso en la UNED, las metodologías usadas, ajustes $y$ avances}

La unidad didáctica, entendida como el material impreso que se realiza específicamente para la UNED, ha pasado por diversas etapas, donde se evidencian metodologías, ajustes y avances que se hacen explícitos hoy. Cruz et al. (2004) sistematizan algunas de ellas:

Primera etapa (1979-1987). Corresponde con el inicio y apertura de la UNED y con la producción de sus primeras unidades didácticas, que seguían los lineamientos de la corriente conductista. Por lo tanto, la producción daba énfasis a los objetivos específicos, a los ejercicio de autoevaluación, los cuales deben permitir al estudiante autoevaluar el logro de cada uno de esos objetivos, al final del texto el autor anota las respuestas a cada uno de ellos; así como el desarrollo lineal prescriptivo del contenido de acuerdo con los objetivos preestablecidos.

Segunda etapa (1988-1999). Esta puede denominarse "de transición" y se inicia después del Primer Congreso Universitario (1988). Se nota un incremento en los aportes 
de los productores académicos de la UNED para tratar de ofrecer unidades didácticas apropiadas para la educación a distancia y se inicia el cuestionamiento de los principios conductistas, al tomar fuerza la corriente cognitiva. Comienzan a desarrollarse ideas tales como la importancia del aprendizaje significativo y la construcción del conocimiento.

Tercera etapa: (1999-2001). Culmina con el Segundo Congreso Universitario (noviembre 2000), donde se acuerda que la producción académica debe basarse en la integración y colaboración de tecnologías nuevas y tradicionales, de acuerdo con el alcance, las características y las potencialidades de enseñanza y aprendizaje de cada uno de los diferentes medios de comunicación. Las nuevas unidades didácticas se producen tomando en cuenta que son parte de un conjunto integrado de materiales didácticos (texto impreso, audiovisuales, videoconferencias y otros medios tecnológicos) por lo que se les llama unidad didáctica modular y, además, tanto la unidad didáctica como los demás materiales deben basarse en los principios que dicta la misión, visión, proyecto educativo y modelo pedagógico de la UNED.

Díaz (comunicación personal, 9 de agosto, 2010), señaló que después de las etapas anteriores se cuentan dos más. La última corresponde al periodo del 2005-2009 que se caracteriza por incorporar la innovación tecnológica junto con la producción de materiales impresos novedosos.

Pese a esos esfuerzos, el estudiante de la UNED lo que requiere es una unidad didáctica impresa que le brinde los contenidos de la disciplina sin importar la presentación gráfica (Monge et al., 2009). La investigación experimental de Monge et al. (2009) reveló que ni la tipografía, ni la presentación de los párrafos, ni el sexo de los estudiantes, tienen efecto sobre la nota obtenida en un examen. Pero sí existe una preferencia subjetiva por la letra Arial de 12 puntos, espaciado normal entre letras, espacio sencillo entre renglones, numeración lateral de las páginas, fondo blanco para el texto, títulos con negrilla en lugar de letra corriente, y textos con estas seis características: subtítulos, resumen, ideas clave resaltadas, palabras importantes resaltadas con negrita, espacio para hacer anotaciones e imágenes en color. La tipografía y presentación de párrafos no tienen efecto sobre el aprendizaje, pero sí sobre la comodidad al estudiar.

Toda esa evolución en la producción de materiales impresos busca mejorar esta mediación pedagógica con miras al logro de estudiantes con competencias 
para el aprendizaje autónomo e independiente.

\section{Alcances, limitaciones y recomendaciones}

En la Universidad Estatal a Distancia, con un modelo pedagógico centrado en el estudiante, el texto escrito sigue siendo el material didáctico por excelencia, por lo tanto, se debe prestar atención a las actividades didácticas que en ellas se incluyan para que sean capaces de propiciar un aprendizaje autónomo. También deben incorporarse el conjunto de elementos y características identificados por Monge et al. (2009) y por Cruz et al. (2004) que, según opinión de los estudiantes, hacen al texto didáctico como el idóneo para el aprendizaje y la formación académica autónomas en la UNED.

Como parte de las limitaciones para la producción da materiales didácticos se señala la carencia de un perfil de autores con fundamentos teóricos de la educación a distancia y como eje central el estudiante. De allí la importancia de este artículo, que servirá como insumo para la discusión a lo interno de la UNED acerca de la elaboración de materiales didácticos que consideren el modelo pedagógico centrado en el estudiante.

Finalmente, como parte de las recomendaciones se considera de importancia el no escatimar esfuerzos en la investigación permanente que permita generar conocimiento en torno a la temática de los fundamentos teóricos de la educación a distancia y, por ende, de sus mediadores: la unidad didáctica y tecnologías, puesto que se mantienen como medios prioritarios de la unidad didáctica modular, así como el análisis detallado de las implicaciones del modelo pedagógico en la concreción de la unidad didáctica.

También se considera de importancia el abrir espacios que promuevan la reflexión en torno a las competencias laborales básicas de los mediadores pedagógicos en un aprendizaje mediatizado.

Con respecto a la conveniencia de un trabajo de equipo en los niveles de planeamiento y producción del material didáctico, se recomienda incorporar líneas metodológicas entre los actores, para generar acciones que beneficien el proceso. Además, se requiere del fortalecimiento de la práctica de evaluación de la unidad de texto, en aras de lograr la pertinencia del material impreso que demanda el modelo pedagógico que ha adoptado la UNED.

\section{Bibliografía}

Berrocal, V. (2009). Consideraciones para el uso de simulaciones en entornos virtuales como apoyo del aprendizaje de las estrategias de programación de 
computadoras. Innovaciones Educativas, año XI (16): 1-14.

Brenes, O.L. (2009). Potencialidades de Internet como herramienta pedagógica en la educación superior. Innovaciones Educativas, año XI (16), 15-26.

Brocket, R. y R. Hiemtra. (1993). El aprendizaje autodirigido en la educación de adultos. Barcelona, Editorial Paidós.

Cabrera, S., M. Lardone y N. Scattolini. (2004). Experiencia tutorial en educación a distancia. Ier Congreso Virtual latinoamericano de Educación a Distancia. 23 de marzo al 4 de abril. http:// www.ateneonline.net/datos/74_03_Silvia_Cabrera.pdf. Consultado el 4de agosto 2010.

Cardona, G. (2002). Tendencias educativas para el siglo XXI. Educación virtual, online y @learning. Elementos para la discusión. Edutec. Revista Electrónica de Tecnología Educativa. (15) http:// www.uib.es/depart/gte/edutec-e/revelec15/car.htm

Contreras, M. (1999). Educación abierta y a distancia. Alternativa de autoformación para el nuevo milenio. Aprendizaje autónomo. En http://www.salesianoscam. org/opcionpreferencial/viiencuentro/ recursos/guia1/aprendizajeautonomo. doc. Consultado el 7 agosto 2010

Cruz, A., G. D’ Agustino y J. Meza. (2004). Elementos y características del material impreso que favorecen la formación y el aprendizaje a distancia en la UNED. Resultados específicos por escuela y nivel de carrera. XII Congreso Internacional de Tecnología y Educación a Distancia. 3 al 5 de noviembre. Centro de Mejoramiento de los Procesos Académicos (CEMPA).

D’Agostino, G., A. Castillo, C. D'Alton, D. Mora \& R. Umaña. (2006). Uso de apoyos didácticos y actividades de aprendizaje de los estudiantes de la UNED y su enlace con el rendimiento académico. Programa de Investigación: Factores que Inciden en el Rendimiento Académico y la Permanencia de los Estudiantes de la UNED. Proyecto I: Estudio exploratorio. Tema II. UNED, SanJosé, Costa Rica, Vicerrectoría Académica. Centro de Mejoramiento de los Procesos Académicos (CEMPA).

Díaz, L. F. (2010). Importancia de la unidad didáctica como medio para la generación de conocimientos en la UNED. Universidad Estatal a Distancia. Comunicación personal, 9 de agosto.

Duart, J.M. \& A. Sangrà. (2000). Formación universitaria por medio de la web: un modelo integrador para el aprendizaje superior. En: Duart, J.M.; Sangrà, A. (comp.). Aprender en la virtualidad. Barcelona: Gedisa.

García, L. (2000). La educación a distancia; ayer y hoy. En: http://www. quadernsdigitals.net/datos_web/ biblioteca/1_1400/enLinea/10.pdf. consultado el 7 de agosto 2010.

Gutiérrez, B., V. H. Méndez \& M. Montero. (1998). Soluciones colaborativas a problemas del paquete instructivo de la UNED: los aportes de Learning Space, Quorum y Correo electrónico. En IX Congreso Internacional sobre Tecnología y Educación a Distancia. Cooperación Internacional en Educación a Distancia. 2 al 5 de noviembre. San José, Costa Rica, EUNED, 298-310.

Laborí, B. \& I. Oleagordia. (s.f.) Estrategias educativas para el uso de las nuevas tecnologías de la información y comunicación. OEI - Revista Iberoamericana de Educación. http://www.rieoei. org/deloslectores/Labori.PDF 
Trilla, J., B. Gros, F. López y Ma. J. Martín. (2003). La educación fuera de la escuela. Ámbitos no formales y educación social. Barcelona España, Ariel Educación. http://books.google.com/book s?id=LvuH5qYFxDsC\&pg=PA156\&1 $\mathrm{pg}=\mathrm{PA} 156 \& \mathrm{dq}=\mathrm{E} 1+\mathrm{impreso},+\mathrm{un}+\mathrm{me}$ dio+privilegiado + de + ense $\% \mathrm{C} 3 \% \mathrm{~B} 1 \mathrm{an}$ za. \&source $=$ bl\&ots $=$ omeECFc_z\&sig $=5 \mathrm{pKGuSoj7S8C0B4jBHLszuR-UY4}$ $\&$ hl=es\&ei=LzFHTfD0JoG81QfosuA $2 \&$ sa $=X \&$ oi=book_result\&ct $=$ result $\&$ resnum $=7 \&$ ved $=0$ CDUQ6AEwBg\#v $=$ onepage $\& \mathrm{q}=\mathrm{El} \% 20 \mathrm{impreso} \% 2 \mathrm{C} \% 20$ un $\% 20$ medio $\% 20$ privilegiado $\% 20$ $\mathrm{de} \% 20 \mathrm{ense} \% \mathrm{C} 3 \% \mathrm{~B} 1$ anza. $\& \mathrm{f}=$ false

Láscaris, A. (2005). La unidad didáctica modular en la UNED de Costa Rica; estrategias. En: http://e-spacio.uned. es/fez/view.php?pid=bibliuned:19427. Consultado 4 de agosto.

Mayorga, R. (1999). Los desafíos a la universidad latinoamericana en el siglo XXI. Organización de Estados Iberoamericanos (OEI). Revista Iberoamericana de Educación, (21). http://www. rieoei.org/rie21a02.htm

Méndez-Estrada, V. H. et al. (2008). Los modelos pedagógicos centrados en el estudiante: apuntes sobre los procesos de aprendizaje y enseñanza. San José. UNED, Programa de investigación en fundamentos de la educación a distancia. PROIFED.

Méndez- Estrada, V. H., J.M Grandbois \& J. Monge-Nájera. (2004). La enseñanza de conceptos básicos de la célula a través de un laboratorio virtual. XII Congreso Internacional de Tecnología y Educación a Distancia. San José, Costa Rica, noviembre.

Méndez-Estrada, V. H. (1998). Multimedios: la visión de los desarrolladores de proyectos. En Aplicación de nuevas tecnologías en la UNED: evaluación mediante grupos focales con profesionales involucrados. San José, Universidad Estatal a Distancia, Centro de Investigación General (CIG). Informe de investigación

Méndez, V. H. (1999). Incorporación de nuevos elementos al paquete instructivo de la UNED de Costa Rica: el caso del curso Biodiversidad e Inventario de la Naturaleza. Innovaciones Educativas, Año VI, (10), 53-59. San José, Costa Rica, EUNED.

Méndez-Estrada, V. H. \& J. Monge-Nájera. (2004). La enseñanza de los modelos ecológicos de depredación a través de un laboratorio virtual. Universidad Estatal a Distancia. XII Congreso Internacional sobre Tecnología y Educación a Distancia, 3 al 5 de noviembre, San José, Costa Rica.

Méndez Estrada, V. H. \& J. Monge-Nájera. (2006). Opinión de los estudiantes acerca del uso de las tecnologías de la información y comunicación empleadas como parte de la evaluación en el curso de Biología General, II cuatrimestre, 2005. Universidad Estatal a Distancia, Centro de Mejoramiento de los Procesos Académicos (CEMPA). Informe final de investigación.

Méndez-Estrada, V. H. \& H. Aguirre. (2010). Memoria institucional de investigaciones realizadas por distintos centros de investigación de la UNED. Universidad Estatal a Distancia, Vicerrectoría de Investigación, Programa de Investigación en Fundamentos de la Educación a Distancia. Informe de investigación.

Milachay, Y. (2007). Estrategias didácticas para el uso de las TIC en la enseñanza de postgrado. Ier Encuentro internacional de Educación Especial. Universidad Salazar, Instituto de estudios Superiores de Chiapas. 26 al 28 de abril. 
Monge-Nájera, J. \& M. Aguilar. (1999). Lecciones aprendidas de la adaptación de un sitio web de educación a distancia para personas con discapacidad visual. X Congreso Internacional sobre Tecnología y Educación a Distancia. El estudiante de la educación a distancia en la perspectiva de un nuevo milenio. San José, Editorial Universidad Estatal a Distancia.

Monge-Nájera, J., M. Rivas Rossi \& V. H. Méndez-Estrada. (1999). Internet, multimedia and virtual laboratories in a "third world" environment: how we solved the 21 basic problems in the Costa Rican distance education university. $X$ Congreso Internacional sobre Tecnología y Educación a Distancia. El estudiante de la educación a distancia en la perspectiva de un nuevo milenio. San José, Editorial Universidad Estatal a Distancia: 416-428.

Monge-Nájera, J., M. Rivas \& V. H. Méndez-Estrada. (2001). Internet, multimedia and virtual laboratories in a "Third world" environment. The Journal of Open and Distance Learning, Inglaterra, 16 (3), October, 279-290.

Monge-Nájera, J. y V. H. Méndez-Estrada. (2004). Análisis de la primera capacitación a tutores y encargados de cátedra y programa, sobre el uso apropiado de los materiales multimediales y otros componentes de la Unidad Didáctica Modular. Universidad Estatal a Distancia, Centro de Mejoramiento de los Procesos Académicos (CEMPA). Informe final de investigación.

Monge Nájera J. \& V. H., Méndez Estrada. (2007). Ventajas y desventajas de usar laboratorios virtuales en educación a distancia: la opinión del estudiantado en un proyecto de seis años de duración. Revista de Educción 31(1), 91-108. ISSN 0379-7082. San José, Costa Rica, Universidad de Costa Rica. Dirección electrónica http://redalyc.uaemex.mx/redalyc/ pdf/440/44031106.pdf

Monge-Nájera, J., V. H. Méndez-Estrada \& A. Villalobos. (2009). Efecto de la tipografía y presentación de párrafos de textos impresos sobre el aprendizaje a distancia en personas adultas. San José, Costa Rica, EUNED, Cuadernos de Investigación. 1 (1): 43 - 68.

Morales, V. (2006). La universidad latinoamericana actual: necesidad de replantear su misión. Caracas, Venezuela, Saber.ula.ve. Universidad Central de Venezuela, Centro de Estudios e Investigaciones sobre Educación Avanzada (CEISEA). Revista Mundo Universitario, (7). http://www.saber. ula.ve/bitstream/123456789/22144/1/ articulo9.pdf

Oficina de programación curricular. PROCUR. (1992). ¿Cómo elaborar la macroprogramación? San José, material mimeografiado.

Ramírez, C. (2006). La tercera revolución educativa costarricense: memorias de la creación y puesta en marcha de la UNED (1975-1982). San José, Costa Rica, EUNED.

Rodino, A. Ma. (1999). En la Universidad y en la vida. La Competencia de escritura de los adultos en relación con sus prácticas lingüísticas cotidianas. $\mathrm{X}$ Congreso Internacional sobre Tecnología y Educación a Distancia. El estudiante de la educación a distancia en la perspectiva de un nuevo milenio. San José, Editorial Universidad Estatal a Distancia, 535-547.

Universidad Estatal a Distancia, UNED. (2007). Modelo pedagógico de la Universidad Estatal a Distancia. Vicerrectoría Académica, CIDREB, marzo. 